\title{
Inhibition of AMPA receptor trafficking at hippocampal synapses by $\beta$-amyloid oligomers: the mitochondrial contribution
}

\author{
Yanfang Rui, Jiaping Gu, Kuai Yu, H Criss Hartzell, James Q Zheng*
}

\begin{abstract}
Background: Synaptic defects represent a major mechanism underlying altered brain functions of patients suffering Alzheimer's disease (AD) [1-3]. An increasing body of work indicates that the oligomeric forms of $\beta$-amyloid (A $\beta$ ) molecules exert profound inhibition on synaptic functions and can cause a significant loss of neurotransmitter receptors from the postsynaptic surface, but the underlying mechanisms remain poorly understood. In this study, we investigated a potential contribution of mitochondria to A $\beta$ inhibition of AMPA receptor (AMPAR) trafficking.
\end{abstract}

Results: We found that a brief exposure of hippocampal neurons to A $\beta$ oligomers not only led to marked removal of AMPARs from postsynaptic surface but also impaired rapid AMPAR insertion during chemically-induced synaptic potentiation. We also found that $A \beta$ oligomers exerted acute impairment of fast mitochondrial transport, as well as mitochondrial translocation into dendritic spines in response to repetitive membrane depolarization. Quantitative analyses at the single spine level showed a positive correlation between spine-mitochondria association and the surface accumulation of AMPARs. In particular, we found that spines associated with mitochondria tended to be more resistant to $A \beta$ inhibition on AMPAR trafficking. Finally, we showed that inhibition of GSK3 $\beta$ alleviated A $\beta$ impairment of mitochondrial transport, and effectively abolished A $\beta$-induced AMPAR loss and inhibition of AMPAR insertion at spines during CLTP.

Conclusions: Our findings indicate that mitochondrial association with dendritic spines may play an important role in supporting AMPAR presence on or trafficking to the postsynaptic membrane. A $\beta$ disruption of mitochondrial trafficking could contribute to AMPAR removal and trafficking defects leading to synaptic inhibition.

\section{Background}

Alzheimer's disease (AD) often attacks aged populations and is highlighted by progressive loss of memory and cognitive abilities [4]. AD brains exhibit two major pathological hallmarks: extracellular senile plaques containing $\beta$-amyloid aggregates and intracellular neurofibrillary tangles consisting of hyperphosphorylated microtubule-associated tau proteins $[5,6]$. $\beta$-amyloid (A $\beta)$ molecules are generated by proteolytic cleavage of the transmembrane $\beta$-amyloid precursor protein (APP) $[7,8]$. Aggregated $A \beta$ fibrils constitute the core of neuritic plaques and are believed to be a major culprit for neurodegeneration and subsequent cognitive

\footnotetext{
* Correspondence: james.zheng@emory.edu

Departments of Cell Biology and Neurology, Center for Neurodegenerative Diseases, Emory University School of Medicine, Atlanta, GA 30322, USA
}

abnormalities in AD patients [9-11]. Recent studies, however, indicate that $A \beta$ molecules exert adverse effects on neuronal functions independent of cell death. Specifically, soluble $A \beta$ oligomers were found to exert severe inhibition of synaptic functions and plasticity [1,12-14], including impairment of long-term potentiation (LTP) and facilitation of long-term depression (LTD) of central synapses $[15,16]$. Therefore, a better understanding of $A \beta$ inhibition of synaptic functions would provide significant insights into the AD neuropathogenic process, potentially leading to better strategies for prevention and treatment of $\mathrm{AD}$.

A major mechanism to modify synaptic strength is to alter the number, types, or properties of neurotransmitter receptors at the postsynaptic terminal [17-20]. The major ionotropic glutamate receptors involved in 
excitatory synaptic transmission are alpha-amino-3hydroxy-5-methyl-4-isoxazolepropionic acid receptors (AMPARs) and $N$-methyl $D$-aspartate receptors (NMDARs). AMPARs are best studied for their rapid trafficking into and out of the synapse by cycling between intracellular stores and the cell surface during synaptic potentiation and depression, respectively [19-22]. NMDARs, due to their voltage-dependent blockade by $\mathrm{Mg}^{2+}$, are thought to function as a coincidence detector of presynaptic and postsynaptic firing and act as the trigger of LTP. It has been shown that activity-dependent trafficking of NMDARs also plays an important role in synaptic plasticity and its alteration may contribute to neuropsychiatric disorders [23]. There is an increasing body of evidence to show that $A \beta$ molecules, especially soluble $A \beta$ oligomers, exert a negative impact on glutamate receptor trafficking in central synapses, leading to synaptic deficits. For example, soluble $A \beta$ oligomers have been shown to bind to AMPARs [24] or NMDARs [25] to cause their internalization, leading to inhibition of LTP and synaptic activity. However, the precise cellular mechanisms underlying $A \beta$ effects on glutamate receptors remain to be elucidated.

Mitochondria are a vital organelle involved in many, if not all, functions of cells. Not only are mitochondria the main energy source of the cell, but they also serve as a part of intracellular $\mathrm{Ca}^{2+}$ stores and regulate intracellular $\mathrm{Ca}^{2+}$ homeostasis, and most importantly regulate cell apoptosis [26-29]. Mitochondria are mostly produced in the cell body and transported to specific cellular locations of increased energy needs such as synapses. It is clear that synaptic transmission and remodeling require localized mitochondria to generate ATP as well as to control local $\mathrm{Ca}^{2+}$ concentrations [30,31]. While mitochondria are known to accumulate at the presynaptic terminal for neurotransmitter release[32], localization of mitochondria to the postsynaptic terminals has also been demonstrated [33]. Our previous study showed that soluble $\mathrm{A} \beta$ molecules acutely impair mitochondrial movement in cultured hippocampal neurons [34]. We thus speculated that disruption of mitochondrial localization to synapses may exert adverse effects on synaptic functions. In this study, we utilized live-cell imaging to investigate whether soluble $A \beta$ oligomers adversely affect AMPAR trafficking at the postsynaptic terminal and its potential mitochondrial connection. We show that soluble $A \beta$ oligomers caused acute reduction of AMPARs on the spine surface and impaired AMPAR insertion during chemically-induced LTP. Furthermore, A $\beta$ oligomers rapidly impaired mitochondrial transport and translocation into dendritic spines. Our analyses revealed that mitochondrial localization to spines is positively correlated to the presence/insertion of AMPARs on the spine surface. Finally, inhibition of
GSK3 $\beta$ prevented A $\beta$ inhibition of both mitochondrial transport and AMPAR trafficking. Together, these findings indicate that mitochondrial localization to dendritic spines may be important for AMPAR trafficking and acute $A \beta$ impairment of mitochondrial trafficking could contribute to the adverse effects of $A \beta$ on AMPARs at synapse.

\section{Results}

$A \beta$ oligomers decrease surface AMPARs and inhibit TEA induced surface AMPAR increase at dendritic spines

We took advantage of $\mathrm{pH}$-dependent fluorescence emission of pHluorin molecules and expressed a super-ecliptic pHluorin fused to the N-terminus of the AMPAR glutamate receptor 1 (SEP-GluR1) in cultured hippocampal neurons for live-cell imaging of AMPAR trafficking. The strong fluorescence of SEP at $\mathrm{pH} \geq 7.0$ (e.g. extracellular solution: $\mathrm{pH}$ 7.4) allowed us to detect the surface presence and dynamic changes of SEP-GluR1 at the single spine level $[35,36]$. We found that SEPGluR1-expressing neurons exhibited numerous spines along the dendritic processes, many of which exhibited strong SEP-GluR1 fluorescence (Figure 1a, also see Figure S1 in Additional file 1). On the other hand, the dendritic shaft, as well as a substantial number of the spines, displayed a low level of diffuse SEP-GluR1 fluorescence, which was considered as the background signals of unclustered SEP-GluR1. These imaging data are consistent with the notion that spines represent the postsynaptic terminals of excitatory synapses with concentrated glutamate receptors. We confirmed that the strong SEP-GluR1 fluorescence came from surface SEPGluR1 as it was effectively quenched by cell-impermeable acidic buffer (Additional file 1, Figure S1).

Recent studies have shown that long term exposure to A $\beta$ oligomers decreases synaptic AMPAR number and impairs AMPAR trafficking [24,37]. We therefore tested if $A \beta$ oligomers exert any acute effects on surface AMPARs. We prepared an oligomeric A $\beta$ solution (A $\beta$ $O$, see Methods) and western blotting confirmed the presence of dimers and trimers, as well as monomers (Figure 1b). Based on the western blot, the amount of oligomers (dimers and trimers) is less than $10 \%$ of the monomers in this $A \beta$ preparation. We found that a 30 min exposure of hippocampal neurons to $5 \mu \mathrm{M} A \beta-\mathrm{O}$ ( $<500 \mathrm{nM}$ oligomers, based on the western blot) resulted in a marked loss of surface SEP-GluR1 at numerous spines (lower panels in Figure 1a, arrows; see also Figure S2 in Additional file 1), whereas similar exposure to a control saline (HBS) had no effect (upper panels in Figure 1a; see also Figure S2 in Additional file 1). We quantified the numbers of spines exhibiting SEP-GluR1 fluorescence before and after 30 min treatment using an intensity threshold that cut off the baseline fluorescence 

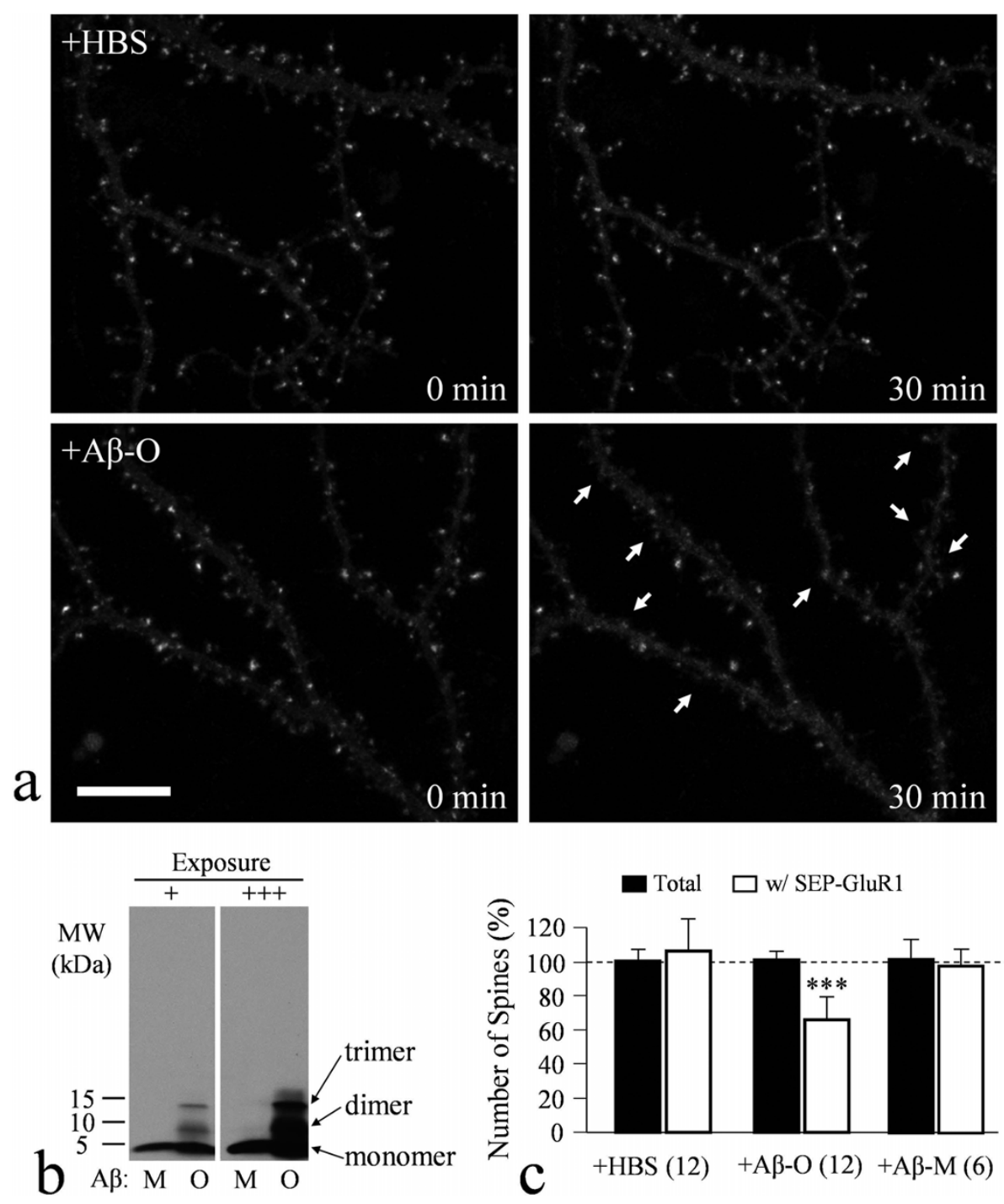

Figure 1 A $\boldsymbol{\beta}$-induced loss of surface AMPARs as revealed by confocal live-cell imaging of SEP-GluR1. (a) Representative images of dendritic regions of cultured hippocampal neurons (DIV21) expressing SEP-GluR1 before and after 30 min exposure to the control saline (HBS, upper panels) or $5 \mu \mathrm{MA} \beta_{1-42}$ solution (lower panels). SEP-GluR1 signals are mostly seen concentrating in dendritic spines. Arrows indicate the spines exhibiting substantial loss of SEP-GluR1 signals after 30 min exposure to A $\beta$. Scale bar: $10 \mu \mathrm{m}$. (b) Western blots showing the existence of $A \beta$ monomers and oligomers in our $A \beta$ preparation. Two different exposures (+ short, +++ long) were shown to ensure that no additional $A \beta$ aggregates in A $\beta-M$ and $A \beta-O$ preparations. (c) Quantitative analysis showing the number of total spines or spines exhibiting strong SEP-GluR1 fluorescence after 30 min exposure to HBS or A $\beta$. The data were normalized against the number before the 30 min exposure with 100\% indicating no change. Triple asterisks: $p<0.0005$ comparing to the corresponding control group (Student's $t$-test).

of dendritic shaft (see Methods and Figure S2 in Additional file 1). Our results show that $A \beta-O$ resulted in $\sim 40 \%$ reduction in the number of spines emitting strong SEP-GluR1 fluorescence, while the total number of spines was not changed (Figure 1c). To test if $A \beta$ oligomers were the ones affecting AMPARs, we prepared an $A \beta$ solution containing only monomers $(A \beta-M$; Figure $1 b)$. Our data showed that $A \beta$ monomers had no effect on SEP-GluR1 signals at dendritic spines (Figure 1c), confirming the notion that $A \beta$ monomers do not affect neuronal viability and functions $[1,38]$. Together, our data show that $A \beta$ oligomers in submicromolar concentration markedly reduce surface AMPARs at postsynaptic terminals.

To study the influence of $A \beta$ oligomers on AMPAR trafficking during synaptic plasticity, we adopted a method to chemically induce LTP (cLTP) by a brief exposure of cells to a potassium channel blocker tetraethylammonium (TEA), which has been shown to robustly elicit AMPAR-dependent LTP in brain slices [39]. We exposed mature (DIV21) hippocampal neurons expressing SEP-GluR1 to $25 \mathrm{mM}$ TEA in a high-calcium 
and low-magnesium environment for $10 \mathrm{~min}$ and examined changes in SEP-GluR1 fluorescence at spines. The potentiation of synaptic efficacy by this cLTP method was confirmed by whole cell patch-clamp recording of both spontaneous and miniature excitatory postsynaptic currents (Additional file 1, Figure S3). We found that a 10 min TEA exposure resulted in a marked increase in the number of spines exhibiting strong SEP-GluR1 fluorescence (Figure 2a, arrows; see also Figure S1 in Additional file 1). Quantification of the number of spines exhibiting bright SEP-GluR1 fluorescence before and after cLTP showed about 30\% increase, whereas the control saline did not cause any changes (Figure $2 \mathrm{~b}$ ). Pre-treatment of neurons with $5 \mu \mathrm{M} \mathrm{A} \beta$-O for $30 \mathrm{~min}$ eliminated TEA-induced increase in spines exhibiting bright SEP-GluR1 fluorescence (Figure 2b). Therefore, $\mathrm{A} \beta$ oligomers inhibited AMPAR insertion during synaptic potentiation.

\section{$A \boldsymbol{\beta}$ oligomers acutely inhibit mitochondria trafficking} Many mitochondria in neurons display microtubuledependent fast movement in both axonal and dendritic processes, which could be acutely impaired by soluble A $\beta$ molecules $[34,40]$. Here we further confirmed that the oligomeric form of $A \beta$ exerted acute impairment of fast mitochondrial movement in hippocampal neurons (Figure 3), whereas A $\beta$ monomers had no effect (Additional file 1, Figure S4). Mitochondria also translocate into dendritic protrusions (filopodia and spines) in response to neuronal activity, which may play a role in

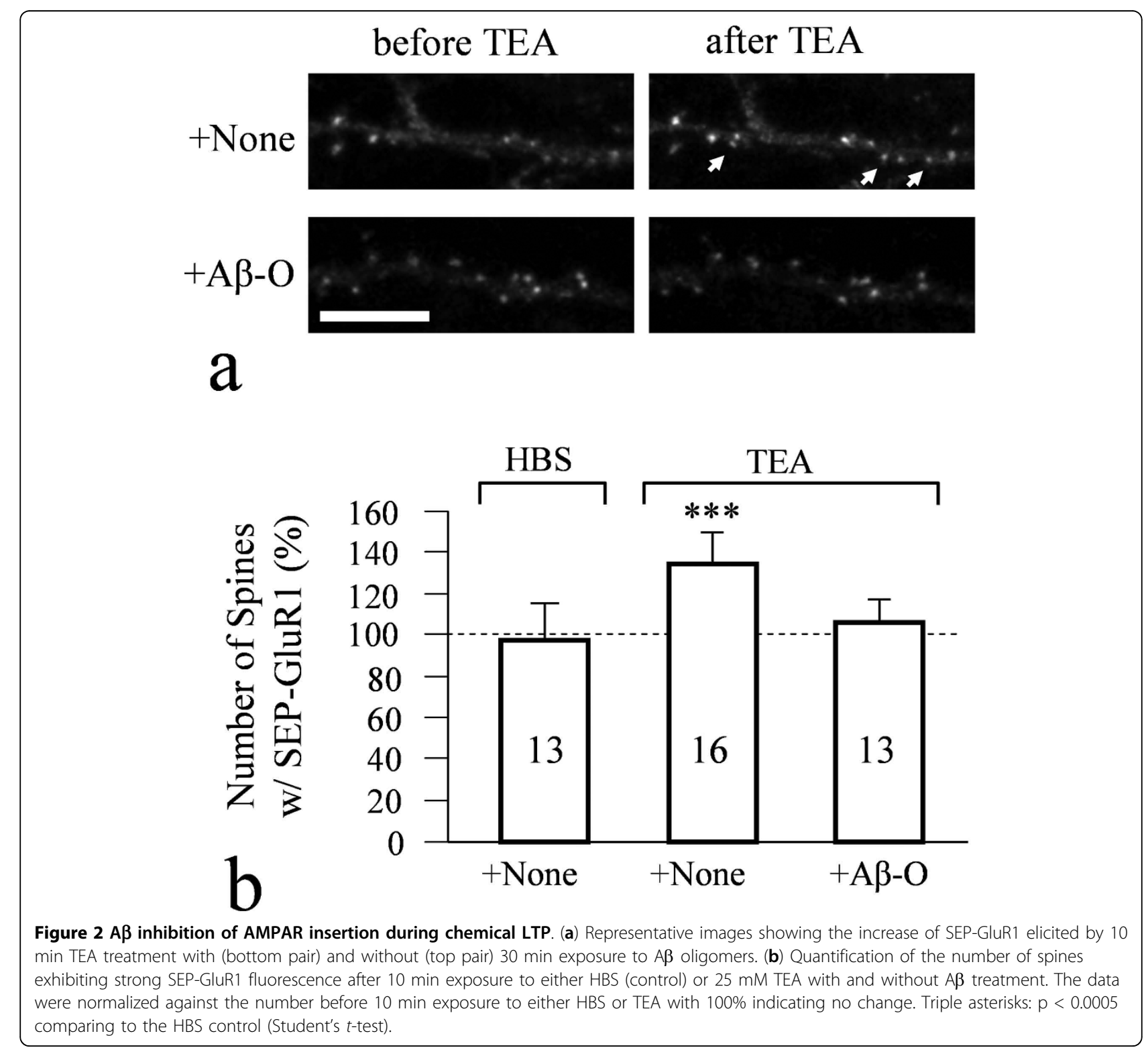



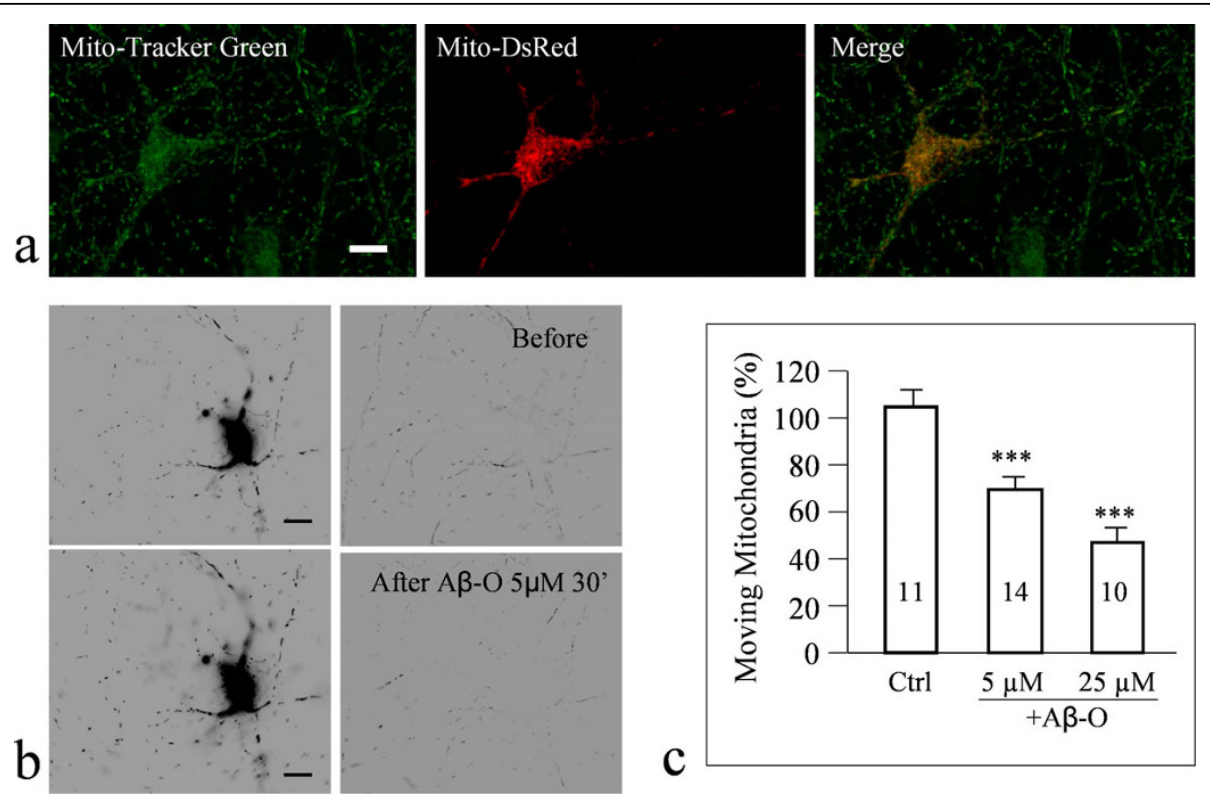

Figure 3 Acute impairment of mitochondrial movement by $A \beta$ oligomers as revealed by time-lapse imaging of Mito-DsRed expressing neurons. (a) Representative images of a hippocampal neuron expressing Mito-DsRed (red), which was also stained by MitoTracker (green). The merged image (the $3^{\text {rd }}$ panel) shows that Mito-DsRed and MitoTracker signals are perfectly colocalized in this transfected neuron, whereas MitoTracker also labeled many more mitochondria in surrounding cells. (b) Representative images from time-lapse sequences before and after 30 min exposure to $A \beta_{1-42}$ oligomers. The left panels represent a snapshot of the mitochondrial distribution in a Mito-DsRed-expressing neuron. The right panels represent the movement traces of mitochondria derived from 5-min time-lapse sequences. The movement traces were generated from the time-lapse sequence using Zprojection followed by division against the first frame using ImageJ software [34]. (c) Quantitative analysis showing the number of moving mitochondria after 30 min treatment with the control saline and different concentration of $A \beta-O$. The data were normalized against the number of moving mitochondria before the 30 min treatment with $100 \%$ indicating no change. Triple asterisks:

$p<0.0005$ (comparing to vehicle, Student's t-test).

synapse development and plasticity [33]. We thus examined if $\mathrm{A} \beta$ oligomers also inhibit activity-dependent mitochondrial translocation into dendritic spines. Consistently, we found that repetitive $\mathrm{KCl}$ depolarization caused a significant increase in the number of spines containing mitochondria (Figure 4a). Quantitative analysis showed that the number of spines containing mitochondria almost doubled after repetitive $\mathrm{KCl}$ treatment (Figure 4b). However, $30 \mathrm{~min}$ exposure of the neurons to $5 \mu \mathrm{M} \mathrm{A} \beta-\mathrm{O}$ completely blocked the increase of mitochondrial translocation into spines by repetitive $\mathrm{KCl}$ depolarization (Figure 4b). Repetitive exposure of neurons to the control saline (KRB) with and without $A \beta-O$ in bath did not affect the number of spines containing mitochondria with and without $A \beta$ presence. Therefore, $A \beta$ oligomers appear to impair fast mitochondrial movement as well as trafficking to postsynaptic terminals in response to membrane depolarization.

\section{$A \beta$ inhibition of surface AMPAR trafficking: a potential mitochondrial contribution}

Given that $A \beta$ oligomers similarly inhibited trafficking of both AMPARs and mitochondria, we hypothesized that a close localization/association of mitochondria to spines may be important for the maintenance of AMPARs on the spine surface, as well as for their increase during synaptic potentiation. To test this hypothesis, we performed extensive analysis on the association of mitochondria with spines exhibiting strong SEP-GluR1 fluorescence or ones with weak fluorescence similar to that of dendritic shaft. We hereafter referred to these two types of spines as bright and dim spines, respectively, for our analysis. Here, hippocampal neurons expressing both SEP-GluR1 and Mito-mOrange were examined by live-cell confocal imaging. Merged color images of SEP-GluR1 and Mito-mOrange show that many spines, especially those bright spines, have mitochondria positioned nearby (Figure 5a). To analyze spine-mitochondria association, we developed a scoring system to give each spine a spine-mito score (Figure 5a): 3 = mitochondria inside the spine, 2 = mitochondria in dendritic shaft but spanning across the entire spine base, 1 = mitochondria in dendritic shaft only partially covering the spine base or immediately adjacent to the spine, $0=$ no mitochondria in the vicinity of at least two-spine distance. Our analysis of several hundreds spines showed that, at baseline, bright spines had a significant higher spine-mito score than dim spines 

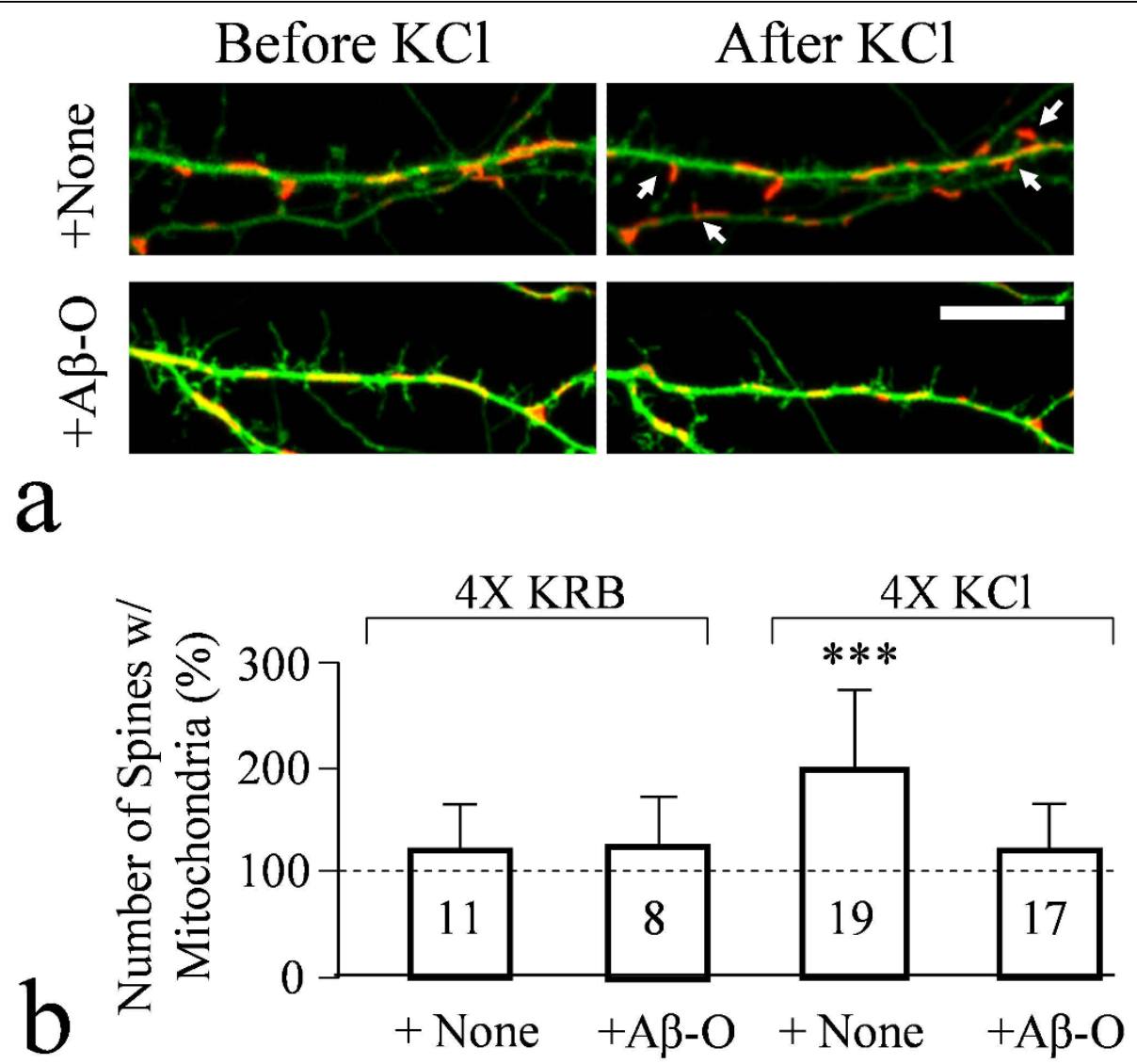

Figure $4 \mathrm{~A} \boldsymbol{\beta}$ impairment of mitochondrial trafficking into spines in response to repetitive $\mathrm{KCl}$ depolarization. (a) Representative images of dendritic segments of hippocampal neurons expressing GFP and Mito-DsRed before and after repetitive KCl depolarization (4X), with (lower pair) and without (upper pair) 30 min exposure to $\mathrm{A} \beta$ oligomers. Arrows indicate spines containing mitochondria after the repetitive $\mathrm{KCl}$ depolarization. Scale bar: $10 \mu \mathrm{m}$. (b) Quantification of the number of spines containing mitochondria after repetitive exposure (4 times) to either KRB (control) or $\mathrm{KCl}$, with and without $A \beta$ treatment. The data are normalized against the number of spines before the repetitive exposure with 100\% indicating no change. Triple asterisks: $p<0.0005$ compared to the control (Student's $t$-test).

(Figure 5b). We also determined the percentage of spines associated with mitochondria (spines with spinemito scores of 1-3) and found that, consistently, more bright spines were associated with mitochondria than dim spines (Figure 5c). These results suggest that mitochondrial association with spines may favor surface presence of AMPARs in spines.

Using the same analysis, we next examined the loss of surface AMPARs induced by acute A $\beta-O$ exposure. Both the spine-mito score and the percentage of spines associated with mitochondria showed a significant difference between bright spines that lost SEP-GluR1 signals and those exhibited no change under $A \beta-O$ exposure (Figure 5b \&5c). Generally, spines exhibiting SEP-GluR1 loss had a lower spine-mito score and less mitochondrial association than those without loss. We next performed similar analysis on the potential association of mitochondria with spines exhibiting AMPAR insertion during cLTP. We found that dim spines exhibiting marked increase in SEP-GluR1 fluorescence had a higher spine-mito score and more mitochondrial association than those without SEP-GluR1 increase (Figure $5 \mathrm{~b} \& 5 \mathrm{c}$ ). Taken together, these data suggest that spines associated with mitochondria tend to favor AMPAR insertion during CLTP and appear to be more resistant to AMPAR loss induced by $A \beta$ oligomers.

Glycogen synthase kinase-3 $\beta$ (GSK3 $\beta$ ) is known to play an important role in $A \beta$ toxicity [41-43] and our previous study showed that inhibition of GSK3 $\beta$ alleviated $\mathrm{A} \beta$ impairment of mitochondrial transport [34]. We hence tested if GSK3 $\beta$ inhibition could also mitigate $A \beta$ inhibition of AMPAR trafficking. Using a specific GSK3 $\beta$ inhibitor SB415286 [44,45], we found that both $A \beta$-induced loss of AMPARs and inhibition of AMPAR insertion during cLTP were largely abolished (Figure 6). These data thus suggest a potentially shared pathway for A $\beta$ impairment of mitochondrial transport and AMPAR trafficking. Taken together, our results suggest that 

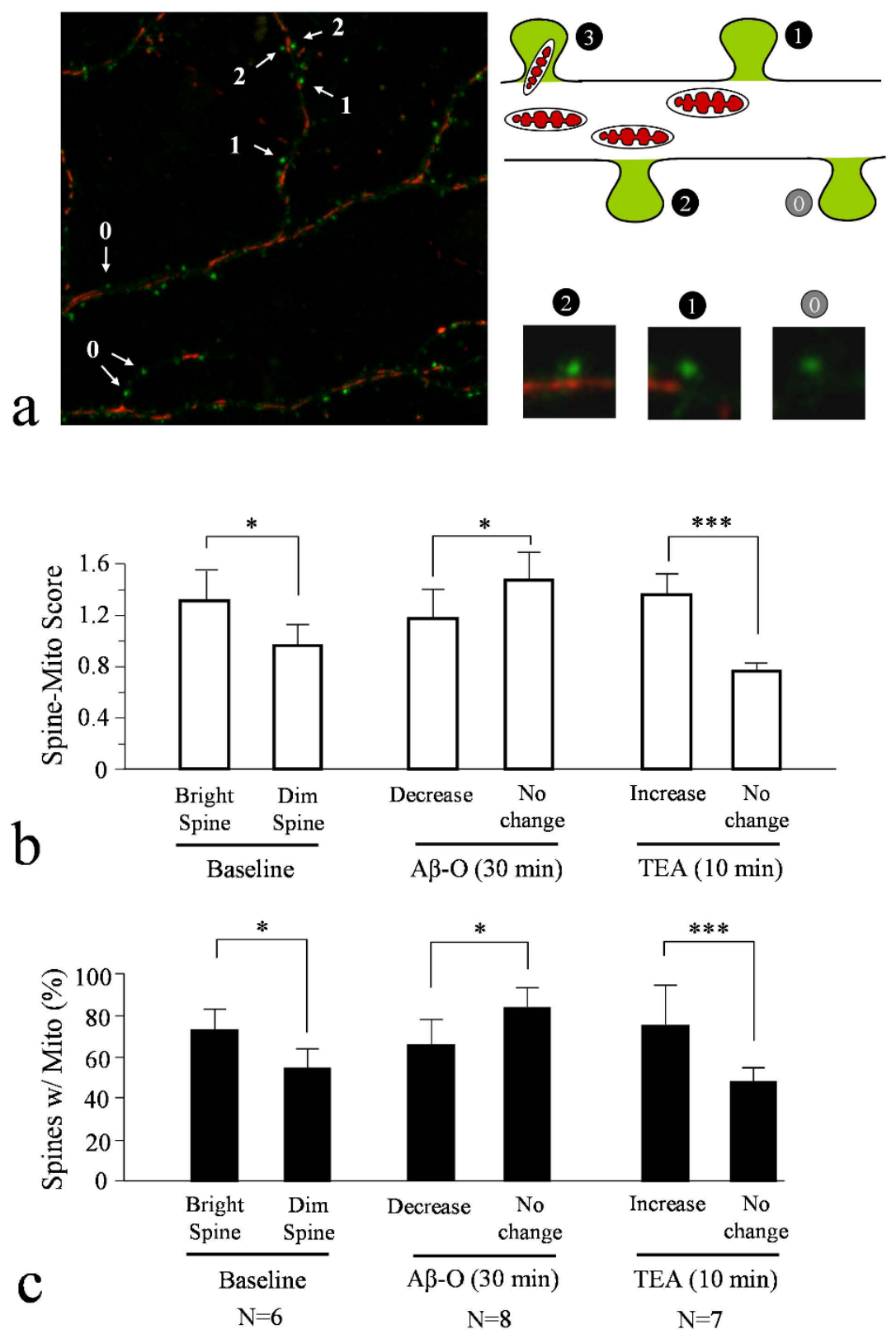

Figure 5 Spine-mitochondria association and its contribution to AMPAR trafficking. (a) A scoring system to assign different spine-mito scores to each spine. The color image on the left shows a merged image of SEP-GluR1 highlighted spines (green) and mitochondria (red). Different scores (0-3) were given to spines depending on their association and proximity to a mitochondrion. The schematic diagram illustrates the scoring criteria, together with examples in magnified view. Analysis of several hundreds of spines from at least three batches of experiments was performed for each condition and data are summarized in the bar graph in (b). Spines receiving spine-mito scores of 1-3 were considered to be associated with mitochondria and used for calculating the percentage of spines associated with mitochondria (c). Single asterisk: $p<0.05$; double asterisk: $p<0.005$; triple asterisks: $p<0.0005$ (comparing to the corresponding control group, Student's t-test).

mitochondrial trafficking and localization to spines may be important for the maintenance of surface AMPARs at spines at the resting state and their increase during plasticity. Acute impairment of mitochondrial trafficking by $A \beta$ oligomers could potentially contribute to or accelerate $A \beta$-induced loss of surface AMPAR and inhibition of AMPAR insertion during synaptic potentiation.

\section{Discussion}

Soluble $A \beta$ oligomers have been shown to impair synaptic functions but the underlying mechanisms remain to be fully understood. At the postsynaptic side of excitatory synapses, $A \beta$-induced internalization of neurotransmitter receptors has been considered to contribute to reduced synaptic strength, but how $\mathrm{A} \beta$ oligomers reduce 

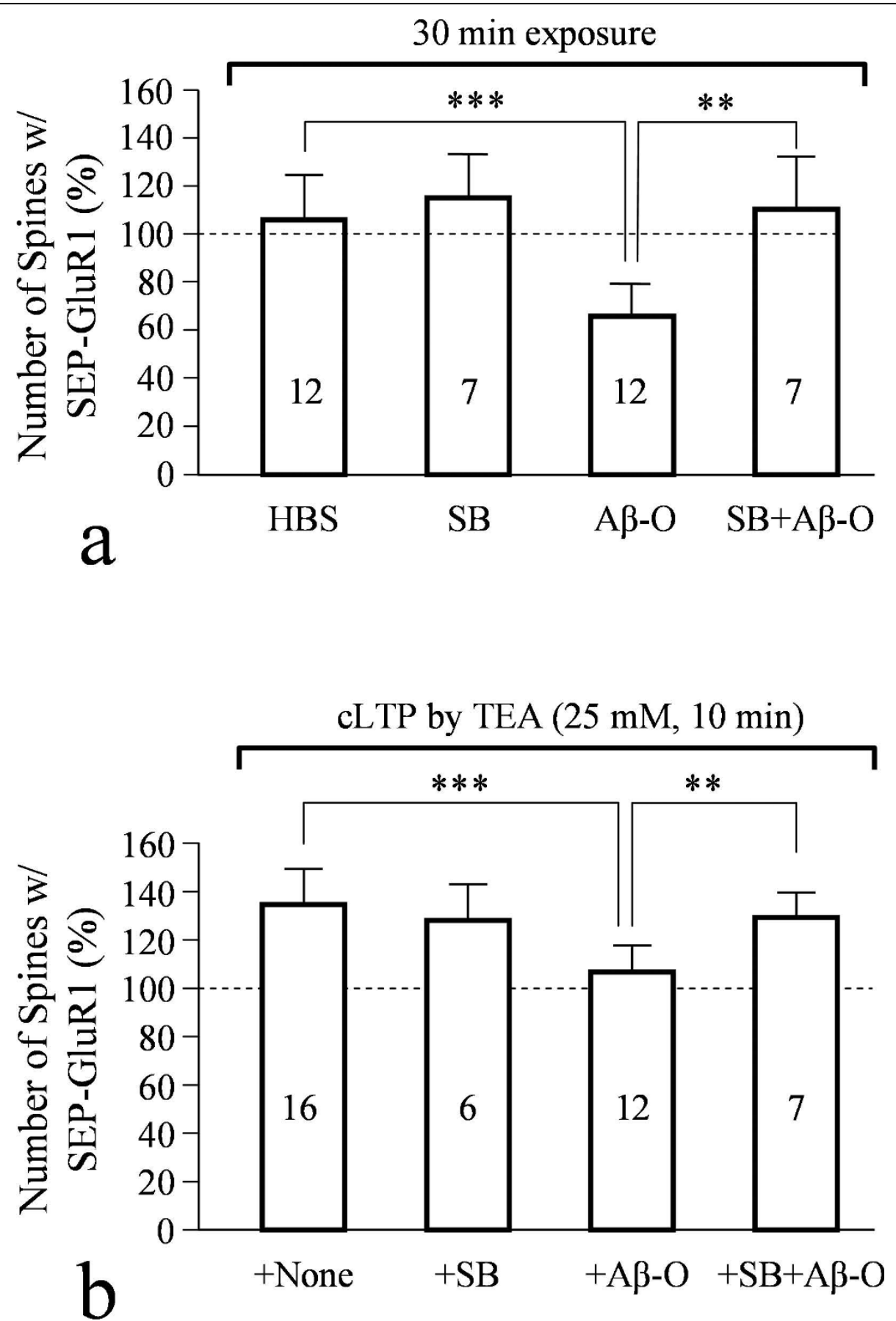

Figure $\boldsymbol{6}$ Involvement of GSK3 $\boldsymbol{\beta}$ in A $\boldsymbol{\beta}$ impairment of AMPAR trafficking. Inhibition of GSK3 $\beta$ by a specific inhibitor SB415286 abolished both $A \beta$-induced loss of AMPARs from spine surface (a) and A $\beta$-inhibition of AMPAR insertion during chemical LTP (b) as revealed by SEP-GluR1 imaging. Double asterisks: $p<0.005$; triple asterisks: $p<0.0005$ (comparing to the control, Student's $t$-test).

surface receptors is unclear. In this study, we used livecell imaging to investigate the acute effects of soluble $A \beta$ molecules on AMPAR trafficking at the postsynaptic terminal and the potential contribution of mitochondria. This study was partially inspired by our previous findings that soluble $A \beta$ molecules acutely inhibit mitochondrial movement in hippocampal neurons, independent of cell death and other drastic alternations of cellular structures [34]. Given that mitochondria are a crucial organelle for energy supply and intracellular $\mathrm{Ca}^{2+}$ regulation, impaired mitochondrial movement could disrupt their proper localization to synaptic sites, 
thus contributing to synaptic deficits elicited by $A \beta$ molecules. Taking advantage of the $\mathrm{pH}$-dependent fluorescence emission of SEP-GluR1, we were able to quantitatively analyze surface AMPARs, their trafficking during CLTP, and the effects of $\mathrm{A} \beta$ oligomers at single spine level. Such an imaging-based approach has allowed us to perform detailed analysis of changes associated with individual spine. For instance, we were able to show that $A \beta$-induced removal of surface AMPARs was not a consequence of spine loss, thus supporting a relatively direct action of $A \beta$ on AMPAR trafficking [24]. Furthermore, when combined with mitochondrial imaging, we were able to reveal a positive correlation between spine localization of mitochondria and AMAPR trafficking. It is quite intriguing to see that local presence of mitochondria appears to favor AMPAR insertion during synaptic potentiation and make them less prone to $A \beta$ inhibition.

While our findings on $A \beta$-induced removal of surface AMPARs and inhibition of insertion during synaptic potentiation were based on imaging of exogenously expressed SEP-GluR1, we have performed surface staining using an anti-GluR1 antibody and confirmed the live imaging results (unpublished results). Furthermore, our results are consistent with previous studies employing electrophysiology, immunostaining, and live-cell imaging in which $A \beta$ was shown to reduce surface AMPARs $[24,37,46]$. A $\beta$-induced reduction of surface AMPARs has been shown to share a common pathway with long term depression (LTD) and to involve $\mathrm{Ca}^{2+}$ signaling through calcineurin for clathrin-mediated endocytosis of AMPARs [24]. On the other hand, how $A \beta$ inhibits AMPAR insertion during cLTP is unclear. Given that AMPAR insertion during LTP depends on $\mathrm{Ca}^{2+}$-dependent exocytosis, A $\beta$-elicited LTD pathway and elevated AMPAR endocytosis could jeopardize LTP signaling cascades to impair AMPAR insertion. While we considered the increase in SEP-GluR1 fluorescence after TEA-cLTP a result of increased AMPAR insertion, our data could not rule out the possibility of decreased AMPAR internalization by TEA. Nonetheless, our study here has provided an intriguing possibility that $A \beta$ impairment of mitochondrial trafficking might contribute to $A \beta$ inhibition on AMPARs. Localization of mitochondria to both pre- and post-synaptic terminals has been observed and likely plays a crucial role for synaptic transmission and remodeling [31-33,47,48]. The rapid inhibition of mitochondrial movement observed previously $[34,40]$ could potentially disrupt the synaptic localization of mitochondria to adversely affect synaptic functions. Indeed we found that a brief exposure of hippocampal neurons to $A \beta$ oligomers inhibited mitochondrial translocation into spines induced by repetitive membrane depolarization.
Based on our correlation analysis, the lack of mitochondrial association appears to facilitate the inhibition of AMPAR trafficking by $A \beta$ oligomers.

How do mitochondria contribute to AMPAR trafficking? Potentially, the local production of ATP by mitochondria is required for vesicular fusion and insertion of AMPARs to the postsynaptic surface. Mitochondria could also be involved in local regulation of intracellular $\mathrm{Ca}^{2+}$ concentrations that are crucial for numerous synaptic activities including synaptic transmission, LTP and LTD, and endo/exocytotic trafficking of membrane proteins. In particular, both LTP and LTD depend on $\mathrm{Ca}^{2+}$ signaling to control synaptic receptor trafficking: the former requires a high $\mathrm{Ca}^{2+}$ elevation for activating CaMKII and downstream effectors for AMPA insertion whereas the latter needs small $\mathrm{Ca}^{2+}$ signals to activate calcineurin phosphatase for AMAPR removal from the surface $[20,49,50]$. The lack of mitochondria at the postsynaptic terminal could alter local $\mathrm{Ca}^{2+}$ signals to favor the LTD pathway for AMPAR removal [24], thus impeding the LTP-induced AMPAR insertion. Certainly, many other synaptic activities, such as ATP-driven ion pumps and local protein synthesis could also depend on the local presence of mitochondria, which could be disrupted by $A \beta$ oligomers. While $A \beta$ disruption of mitochondrial trafficking and localization to synapses might not directly or solely cause AMPAR trafficking defects, it could significantly contribute to postsynaptic defects in coordination and synergy with other $A \beta$-elicited events (e.g. $A \beta$ induced internalization of synaptic receptors). While direct evaluation of this mitochondrial hypothesis requires selective disruption of mitochondrial localization to spines or of specific mitochondrial function(s) at spines, our findings that inhibition of GSK3 $\beta$ mitigate $A \beta$ impairment of trafficking of both AMPAR and mitochondria suggest that these two events could be linked in contributing to $A \beta$-induced synaptic inhibition.

In conclusion, our studies showed that soluble $A \beta$ oligomers exert acute inhibition on the trafficking of both mitochondria and synaptic receptors. The postsynaptically localized mitochondria appear to be important for the maintenance of AMPARs on postsynaptic surface as well as for AMPAR insertion during synaptic potentiation. Intriguingly, our correlation analysis suggests that impairment of mitochondrial trafficking might contribute to the adverse effects of $\mathrm{A} \beta$ oligomers on AMPARs on the postsynaptic surface. Future studies that employ selective targeting of mitochondrial movement could provide more definite answers regarding the precise role of mitochondria in synaptic receptor trafficking, as well as its precise contribution to synaptic defects in $\mathrm{AD}$ brains. 


\section{Methods}

\section{Cell culture and transfection}

Hippocampal neurons from embryonic day 18 rats were obtained according to the method described previously [51]. Dissociated cells were plated in $35 \mathrm{~mm}$ glass bottom culture dishes (Warner Instruments, Hamden, CT) for culture and microscopy. The glass surface was pretreated with $100 \mu \mathrm{g} / \mathrm{ml}$ poly-D-lysine (Sigma, St. Louis, MO) overnight and $\sim 200,000$ cells were plated in each dish in Neurobasal medium containing B27 and Glutamax (Invitrogen). Cells were maintained in a $5 \% \mathrm{CO}_{2}$ incubator at $37^{\circ} \mathrm{C}$, with half of the culture medium replaced with fresh Neurobasal medium every $3 \mathrm{~d}$. Before each imaging experiment, the medium was replaced by Krebs'-Ringer's buffer (KRB, in mM: 150 $\mathrm{NaCl}, 5 \mathrm{KCl}, 2 \mathrm{CaCl}_{2}, 1 \mathrm{MgCl}_{2}, 10$ glucose, and 10 HEPES, pH 7.4) [52] or HEPES-buffered solution (HBS, in mM: $140 \mathrm{NaCl}, 5 \mathrm{KCl}, 2 \mathrm{CaCl}_{2}, 1.5 \mathrm{MgCl}_{2}, 10$ glucose, and 25 HEPES, pH 7.4).

Hippocampal neurons were transfected using CalPhos Mammalian Transfection Kit (Clontech, Mountain View, CA). Neurons plated in $35 \mathrm{~mm}$ culture dishes at different days in vitro (DIV) were used depending on the experiments. Typically, we transfected the cells several days before the imaging experiments to allow the expression of various GFP-fusion or mutant proteins. For experiments on mitochondrial transport, we typically transfected the neurons at DIV6-7 and performed imaging on DIV8-9. For $\mathrm{KCl}$ depolarization experiments, the transfection was performed on DIV12-13 and followed by imaging on DIV14-15. For imaging studies on AMPARs, the transfection was performed on DIV13-14 followed by imaging on DIV21-22 when mature synaptic connections had been formed. The DNA constructs for transfection were prepared by plasmid maxi kit (Qiagen, Valencia, CA). The following constructs were used: Mito-DsRed and Mito-GFP (generously provided by Dr. Zheng Li at NIH/NIMH), pCi-SEP-GluR1 (a gift from Dr. Roberto Malinow at University of California at San Diego), EGFP-C1 and mOrange (Clontech). To create Mito-mOrange, the mOrange coding sequence was subcloned into Mito-GFP vector with green fluorescent protein (GFP) sequence excised.

\section{$A \beta$ preparation and treatment}

We followed the previously published method to prepare $A \beta$ oligomers for our experiments ( $A \beta-O$ solution) [53]. A $\beta_{1-42}$ was purchased from American Peptide Company Inc (Sunnydale, CA) and dissolved in hexafluoro-2-propanol (HFIP) and aliquoted to microfuge tubes. HFIP was subsequently removed by evaporation in a speed-vacuum and desiccated $A \beta$ aliquots were stored at $-20^{\circ} \mathrm{C}$. To make $\mathrm{A} \beta$ oligomer solution, each
$\mathrm{A} \beta_{1-42}$ aliquot was dissolved in DMSO to make a $5 \mathrm{mM}$ stock solution. The solution was diluted to $100 \mu \mathrm{M}$ with $\mathrm{KRB}$ and kept at $4^{\circ} \mathrm{C}$ for $24 \mathrm{hr}$ before use. To make an $A \beta$ solution containing only monomers (A $\beta-M$ solution), $\mathrm{A} \beta_{1-42}$ was directly dissolved in $\mathrm{ddH} 2 \mathrm{O}$ at $1 \mathrm{mM}$, diluted to $100 \mu \mathrm{M}$ with $\mathrm{KRB}$, and incubated at $37^{\circ} \mathrm{C}$ for $7 \mathrm{~d}$. Afterwards, the $\mathrm{A} \beta$ solution was centrifuged at $14,000 \mathrm{rpm}$ for $60 \mathrm{~min}$ to remove $A \beta$ fibrils. The supernatant was collected and passed through a $100 \mathrm{KD}$ molecular weight cut-off (MWCO) Amicon centrifugal filter (Millipore) to further remove any large $A \beta$ aggregates. Western blotting showed that this method produced only $\mathrm{A} \beta$ monomers (Figure $1 \mathrm{~b}$ ). The concentration of $\mathrm{A} \beta$ monomers in solution was determined using Bradford Protein Assay (Bio-Rad) and adjusted to the same concentration of $A \beta-O$ solution.

Bath application of $\mathrm{A} \beta$ was achieved through a twostep dilution procedure. First, the $A \beta$ stock solution was diluted in KRB to twice the designated concentration ( $2 \times$ working stock). The $2 \times$ working stock solution was then gently added to and mixed with the bath saline of the cells in an equal volume to reach the desired final concentration. In a typical experiment, $1 \mathrm{ml}$ of the $2 \times$ stock solution was added to $1 \mathrm{ml}$ of the bath solution in the culture/imaging dish on the microscope stage.

\section{Western blotting to detect $\boldsymbol{A} \boldsymbol{\beta}$ molecules}

We used 4G8 anti-A $\beta$ antibody (Signet, Dedham, MA) to perform western blotting to detect different forms of $\mathrm{A} \beta$ in our preparation. $80 \mathrm{ng} \mathrm{A} \beta$ samples were added to sample buffer with $50 \mathrm{mM}$ DTT and heated at $85^{\circ} \mathrm{C}$ for $2 \mathrm{~min}$. Samples were loaded and fractioned by PAGE on 10-20\% Tris-Tricine gel (Invitrogen) and subsequently transferred to nitrocellulose membranes. The membrane was boiled for $10 \mathrm{~min}$ in PBS and blocked with 5\% nonfat dry milk in TBS with $0.05 \%$ Tween-20 (TBST) for 1 $\mathrm{h}$ at room temperature. The membrane was then incubated with 4 G8 antibody (1:1000) in blocking buffer overnight at $4^{\circ} \mathrm{C}$. Bound antibodies were detected by HRP-conjugated secondary antibody, visualized by chemiluminescence using ECL (Thermo Scientific, Rockford, IL), and quantified using the gel analysis routine of ImageJ software (NIH).

\section{Live cell imaging of mitochondrial movement}

Fluorescent time-lapse recordings were performed on an inverted microscope (TE2000, Nikon) using a 40× N.A. 1.3 S-Fluor oil immersion objective with identical settings between the control and experimental groups. Time-lapse images were captured with a CCD camera (SensiCam QE, Cooke Scientific) using the IPLab imaging software (BD Biosciences). For imaging of mitochondrial transport, we typically recorded neurons at a 
sampling rate of one frame every $5 \mathrm{~s}$ for $5 \mathrm{~min}$, with the CCD exposure at $50 \mathrm{~ms}$ exposure and $2 \times 2$ binning. For each experiment, a population of neurons was imaged for a $5 \mathrm{~min}$ control period before the application of $\mathrm{A} \beta$ molecules, followed by another $5 \mathrm{~min}$ time-lapse recording at $30 \mathrm{~min}$ after $\mathrm{A} \beta$ application. All the experiments were performed on the microscope stage with the $35 \mathrm{~mm}$ dish housed in a temperature controlled chamber (Warner Instruments, New Haven, CT) with the temperature set at $\sim 35^{\circ} \mathrm{C}$. Quantification of moving mitochondria was done by simply counting the number of moving mitochondria in each $5 \mathrm{~min}$ time-lapse sequence. A moving mitochondrion was defined as one that moved more than a distance of twice its length over the $5 \mathrm{~min}$ period. Since no change in the total mitochondrial number was observed [34], we normalized the number of moving mitochondria in the 5-min sequence against that before the $A \beta$ application. A value of $100 \%$ indicates that same numbers of moving mitochondria were observed in both recording periods.

\section{Confocal live-cell imaging on mitochondrial association with dendritic spines and AMPAR trafficking}

A Nikon C1 confocal on TE300 inverted microscope, together with a $60 \times$ N.A.1.4 Plan Apo oil immersion objective, was used for imaging. To be able to examine all the spines at different focusing planes of a dendritic segment, a z-stack of 10-12 images was taken on a selected dendritic region followed by maximal intensity projection to generate the 2-D image. For experiments on $\mathrm{KCl}$-stimulated mitochondria translocation into spines, two-channel confocal imaging was performed on neurons expressing EGFP and Mito-DsRed at DIV14-15. To stimulate mitochondrial translocation into spines, we used a previously described method of repetitive membrane depolarization by $\mathrm{KCl}$ [33]. Here, $90 \mathrm{mM} \mathrm{NaCl}$ of normal KRB was replaced with $90 \mathrm{mM} \mathrm{KCl}$ (hereafter referred to as $\mathrm{KCl}-\mathrm{KRB}$ ) for membrane depolarization. We performed 4 times of $\mathrm{KCl}-\mathrm{KRB}$ exposure, each exposure for $3 \mathrm{~min}$ and separated by $10 \mathrm{~min}$ recovery in normal KRB. The same neurons were imaged before and one hour after the $4 \times \mathrm{KCl}$ stimulation to examine the association of mitochondria with spines.

Similar confocal imaging was performed on hippocampal neurons expressing SEP-GluR1 to study AMPAR trafficking. Since SEP-GluR1 only emits strong fluorescence on cell surface and forms clusters as endogenous AMPARs at postsynaptic terminals, we used an intensity threshold that cut off the diffuse SEP-GluR1 fluorescence of dendritic shaft (considered as background) to select postsynaptic receptor clusters that emitted substantial SEP-GluR1 signals, followed by quantification of their number. Both thresholding and quantification were done using ImageJ software. To examine the effect of A $\beta$ molecules on surface AMPAR clusters, we acquired images of the same dendritic region before and after $A \beta$ exposure, followed by same thresholding and quantification to determine the change in the number of spines with SEP-GluR1 signals. For AMPAR insertion during synaptic potentiation, we used a method involving a brief exposure of cells to a potassium channel blocker tetraethylammonium (TEA) to chemically induce potentiation (cLTP) [39]. Here, we stimulated mature (DIV21) hippocampal neurons expressing SEP-GluR1 with 25 $\mathrm{mM}$ TEA in a high-calcium and low-magnesium solution (in mM: $140 \mathrm{NaCl}, 5 \mathrm{KCl}, 5 \mathrm{CaCl}_{2}, 0.1 \mathrm{MgCl}_{2}, 10$ glucose, and 25 HEPES, 25 TEA, pH 7.4) for $10 \mathrm{~min}$. Confocal live-cell imaging on the same dendritic regions was performed before and after the stimulation to detect changes in SEP-GluR1 fluorescence. Similar thresholding and quantification were done on the two images (before and after cLTP induction) to quantify the change in the number of spines with SEP-GluR1 signals. For A $\beta$ effects on AMPAR insertion during cLTP, we pre-treated the neurons with $A \beta$ oligomers for 30 min before the cLTP induction by TEA.

\section{Electrophysiology}

Conventional whole cell patch-clamp recordings were performed on the cell body of pyramidal hippocampal neurons with voltage-clamped at $-70 \mathrm{mV}$ using an EPC-7 patch-clamp amplifier (HEKA Instruments Inc., Bellmore, NY). Fire-polished borosilicate glass patch pipettes had a resistance of 3-5 $\mathrm{M} \Omega$. Experiments were conducted at room temperature $\left(20-24^{\circ} \mathrm{C}\right)$. Since the liquid junction potentials were small $(<2 \mathrm{mV})$, no correction was made. The standard pipette solution contained $(\mathrm{mM}): 147 \mathrm{KCl}, 2 \mathrm{KH}_{2} \mathrm{PO}_{4}, 5$ Tris- $\mathrm{HCl}, 2$ EGTA, 10 HEPES, 4 Mg-ATP, pH 7.3 adjusted with $\mathrm{KOH}$, and osmolarity at 310-320 $\mathrm{mOsmol}^{-1}$. The extracellular recording solution contained $(\mathrm{mM}): 128 \mathrm{NaCl}$, $5 \mathrm{KCl}, 2 \mathrm{CaCl}_{2}, 1 \mathrm{MgCl}_{2}, 25 \mathrm{HEPES}, 30$ glucose, 0.1 picrotoxin, $\mathrm{pH} 7.3$ with $\mathrm{NaOH}$, and osmolarity at 300$310 \mathrm{mOsmol}^{-1}$. For miniature EPSCs, $0.5 \mu \mathrm{M}$ tetrodotoxin (TTX) was added to the extracellular recording solution. To induce synaptic potentiation, a TEA solution (in mM: $80 \mathrm{NaCl}, 20 \mathrm{KCl}, 2 \mathrm{CaCl}_{2}, 25 \mathrm{TEA}, 25$ HEPES, 30 glucose, pH7.3 and $315 \mathrm{mOsmol}^{-1}$ ) was perfused to the neurons. We typically recorded for 5-10 min before and after $10 \mathrm{~min}$ TEA treatment (25 mM). During the TEA treatment, the patch-clamp amplifier was switched to the current clamp mode with the current set to zero for maximal synaptic stimulation. The cell was re-clamped at $-70 \mathrm{mV}$ after TEA washout. Recorded EPSCs were filtered at $2 \mathrm{kHz}$ before the analysis and presentation. 
Additional file 1: The additional file 1 contains supplemental figures S1-S4.

\section{Acknowledgements}

This work is supported in part by research grants from National Institutes of Health to JQZ (AG029596, GM083889, and GM084363) and HCH (EY014852 and GM60448), as well as by a Pilot award to JQZ from Emory Alzheimer's Disease Research Center (ADRC, P50 AG025688)

\section{Authors' contributions}

YR performed a majority of the experiments and analyses, and wrote the first draft of the manuscript. JG helped with live imaging and analysis. KY of Hartzell lab did the electrophysiological recordings and $\mathrm{HCH}$ provided feedback on the manuscript. JQZ designed, planned, guided the project, as well as did some imaging experiments and writing. All authors have read and approved the final manuscript.

\section{Competing interests}

The authors declare that they have no competing interests.

Received: 5 January 2010 Accepted: 26 March 2010

Published: 26 March 2010

\section{References}

1. Shankar GM, Li S, Mehta TH, Garcia-Munoz A, Shepardson NE, Smith I, Brett FM, Farrell MA, Rowan MJ, Lemere CA, et al: Amyloid-beta protein dimers isolated directly from Alzheimer's brains impair synaptic plasticity and memory. Nat Med 2008, 14:837-842.

2. Lassmann $H$, Fischer $P$, Jellinger $K$ : Synaptic pathology of Alzheimer's disease. Ann N Y Acad Sci 1993, 695:59-64.

3. Blennow K, Bogdanovic N, Alafuzoff I, Ekman R, Davidsson P: Synaptic pathology in Alzheimer's disease: relation to severity of dementia, but not to senile plaques, neurofibrillary tangles, or the ApoE4 allele. J Neural Transm 1996, 103:603-618.

4. Selkoe DJ, Schenk D: Alzheimer's disease: molecular understanding predicts amyloid-based therapeutics. Annu Rev Pharmacol Toxicol 2003, 43:545-584

5. Dickson DW: Neuropathological diagnosis of Alzheimer's disease: a perspective from longitudinal clinicopathological studies. Neurobiol Aging 1997, 18:S21-26.

6. Selkoe DJ: Folding proteins in fatal ways. Nature 2003, 426:900-904.

7. Glenner GG, Wong CW: Alzheimer's disease: initial report of the purification and characterization of a novel cerebrovascular amyloid protein. Biochem Biophys Res Commun 1984, 120:885-890.

8. Mattson MP: Pathways towards and away from Alzheimer's disease. Nature 2004, 430:631-639.

9. Delacourte A, Buee L: Tau pathology: a marker of neurodegenerative disorders. Curr Opin Neurol 2000, 13:371-376.

10. Hardy J: Amyloid, the presenilins and Alzheimer's disease. Trends Neurosci 1997, 20:154-159.

11. Selkoe DJ: Translating cell biology into therapeutic advances in Alzheimer's disease. Nature 1999, 399:A23-31.

12. Walsh DM, Klyubin I, Fadeeva JV, Cullen WK, Anwyl R, Wolfe MS, Rowan MJ, Selkoe DJ: Naturally secreted oligomers of amyloid beta protein potently inhibit hippocampal long-term potentiation in vivo. Nature 2002, 416:535-539.

13. Wang HW, Pasternak JF, Kuo H, Ristic H, Lambert MP, Chromy B, Viola KL, Klein WL, Stine WB, Krafft GA, Trommer BL: Soluble oligomers of beta amyloid (1-42) inhibit long-term potentiation but not long-term depression in rat dentate gyrus. Brain Res 2002, 924:133-140.

14. Lacor PN, Buniel MC, Chang L, Fernandez SJ, Gong Y, Viola KL, Lambert MP, Velasco PT, Bigio EH, Finch CE, et al: Synaptic targeting by Alzheimer'srelated amyloid beta oligomers. J Neurosci 2004, 24:10191-10200.

15. Selkoe DJ: Alzheimer's disease is a synaptic failure. Science 2002, 298:789-791.

16. Kim JH, Anwyl R, Suh YH, Djamgoz MB, Rowan MJ: Use-dependent effects of amyloidogenic fragments of (beta)-amyloid precursor protein on synaptic plasticity in rat hippocampus in vivo. J Neurosci 2001, 21:1327-1333.
17. Malenka RC: Synaptic plasticity and AMPA receptor trafficking. Ann N Y Acad Sci 2003, 1003:1-11.

18. Luscher C, Xia H, Beattie EC, Carroll RC, von Zastrow M, Malenka RC, Nicoll RA: Role of AMPA receptor cycling in synaptic transmission and plasticity. Neuron 1999, 24:649-658.

19. Collingridge GL, Isaac JT, Wang YT: Receptor trafficking and synaptic plasticity. Nat Rev Neurosci 2004, 5:952-962.

20. Malinow R, Malenka RC: AMPA receptor trafficking and synaptic plasticity. Annu Rev Neurosci 2002, 25:103-126.

21. Bredt DS, Nicoll RA: AMPA receptor trafficking at excitatory synapses. Neuron 2003, 40:361-379.

22. Song I, Huganir RL: Regulation of AMPA receptors during synaptic plasticity. Trends Neurosci 2002, 25:578-588.

23. Lau CG, Zukin RS: NMDA receptor trafficking in synaptic plasticity and neuropsychiatric disorders. Nat Rev Neurosci 2007, 8:413-426.

24. Hsieh H, Boehm J, Sato C, Iwatsubo T, Tomita T, Sisodia S, Malinow R: AMPAR removal underlies Abeta-induced synaptic depression and dendritic spine loss. Neuron 2006, 52:831-843.

25. Snyder EM, Nong Y, Almeida CG, Paul S, Moran T, Choi EY, Nairn AC, Salter MW, Lombroso PJ, Gouras GK, Greengard P: Regulation of NMDA receptor trafficking by amyloid-beta. Nat Neurosci 2005, 8:1051-1058.

26. Young KW, Bampton ET, Pinon L, Bano D, Nicotera P: Mitochondrial Ca2+ signalling in hippocampal neurons. Cell Calcium 2008, 43:296-306.

27. Shaw JM, Nunnari J: Mitochondrial dynamics and division in budding yeast. Trends Cell Biol 2002, 12:178-184.

28. Wang X, Schwarz TL: Imaging axonal transport of mitochondria. Methods Enzymol 2009, 457:319-333.

29. Fannjiang Y, Cheng WC, Lee SJ, Qi B, Pevsner J, McCaffery JM, Hill RB, Basanez G, Hardwick JM: Mitochondrial fission proteins regulate programmed cell death in yeast. Genes Dev 2004, 18:2785-2797.

30. Tang Y, Zucker RS: Mitochondrial involvement in post-tetanic potentiation of synaptic transmission. Neuron 1997, 18:483-491.

31. Schuman E, Chan D: Fueling synapses. Cell 2004, 119:738-740.

32. Chang DT, Honick AS, Reynolds IJ: Mitochondrial trafficking to synapses in cultured primary cortical neurons. J Neurosci 2006, 26:7035-7045.

33. Li Z, Okamoto K, Hayashi Y, Sheng M: The importance of dendritic mitochondria in the morphogenesis and plasticity of spines and synapses. Cell 2004, 119:873-887.

34. Rui Y, Tiwari P, Xie Z, Zheng JQ: Acute impairment of mitochondrial trafficking by beta-amyloid peptides in hippocampal neurons. J Neurosci 2006, 26:10480-10487.

35. Sankaranarayanan S, De Angelis D, Rothman JE, Ryan TA: The use of pHluorins for optical measurements of presynaptic activity. Biophys J 2000, 79:2199-2208.

36. Lin DT, Huganir RL: PICK1 and phosphorylation of the glutamate receptor 2 (GluR2) AMPA receptor subunit regulates GluR2 recycling after NMDA receptor-induced internalization. J Neurosci 2007, 27:13903-13908.

37. Gu Z, Liu W, Yan Z: \{beta\}-Amyloid impairs AMPA receptor trafficking and function by reducing $\mathrm{Ca} 2+/$ calmodulin-dependent protein kinase II synaptic distribution. J Biol Chem 2009, 284:10639-10649.

38. Li S, Hong S, Shepardson NE, Walsh DM, Shankar GM, Selkoe D: Soluble oligomers of amyloid Beta protein facilitate hippocampal long-term depression by disrupting neuronal glutamate uptake. Neuron 2009, 62:788-801.

39. Aniksztejn $L$, Ben-Ari Y: Novel form of long-term potentiation produced by a K+ channel blocker in the hippocampus. Nature 1991, 349:67-69.

40. Hiruma H, Katakura T, Takahashi S, Ichikawa T, Kawakami T: Glutamate and amyloid beta-protein rapidly inhibit fast axonal transport in cultured rat hippocampal neurons by different mechanisms. J Neurosci 2003, 23:8967-8977.

41. Terwel D, Muyllaert D, Dewachter I, Borghgraef P, Croes S, Devijver H, Van Leuven F: Amyloid activates GSK-3beta to aggravate neuronal tauopathy in bigenic mice. Am J Pathol 2008, 172:786-798.

42. Cedazo-Minguez A, Popescu BO, Blanco-Millan JM, Akterin S, Pei JJ, Winblad B, Cowburn RF: Apolipoprotein E and beta-amyloid (1-42) regulation of glycogen synthase kinase-3beta. J Neurochem 2003, 87:1152-1164.

43. Hooper C, Killick R, Lovestone S: The GSK3 hypothesis of Alzheimer's disease. J Neurochem 2008, 104:1433-1439.

44. Coghlan MP, Culbert AA, Cross DA, Corcoran SL, Yates JW, Pearce NJ, Rausch OL, Murphy GJ, Carter PS, Roxbee Cox L, et al: Selective small 
molecule inhibitors of glycogen synthase kinase-3 modulate glycogen metabolism and gene transcription. Chem Biol 2000, 7:793-803.

45. Cross DA, Culbert AA, Chalmers KA, Facci L, Skaper SD, Reith AD: Selective small-molecule inhibitors of glycogen synthase kinase-3 activity protect primary neurones from death. J Neurochem 2001, 77:94-102.

46. Chang EH, Savage MJ, Flood DG, Thomas JM, Levy RB, Mahadomrongkul V, Shirao T, Aoki C, Huerta PT: AMPA receptor downscaling at the onset of Alzheimer's disease pathology in double knockin mice. Proc Natl Acad Sci USA 2006, 103:3410-3415

47. Mattson MP, Gleichmann M, Cheng A: Mitochondria in neuroplasticity and neurological disorders. Neuron 2008, 60:748-766.

48. Sung JY, Engmann O, Teylan MA, Nairn AC, Greengard P, Kim Y: WAVE1 controls neuronal activity-induced mitochondrial distribution in dendritic spines. Proc Natl Acad Sci USA 2008, 105:3112-3116.

49. Lisman J, Schulman H, Cline H: The molecular basis of CaMKII function in synaptic and behavioural memory. Nat Rev Neurosci 2002, 3:175-190.

50. Winder DG, Sweatt JD: Roles of serine/threonine phosphatases in hippocampal synaptic plasticity. Nat Rev Neurosci 2001, 2:461-474

51. Banker GA, Cowan WM: Rat hippocampal neurons in dispersed cell culture. Brain Res 1977, 126:397-342.

52. Bacci A, Verderio C, Pravettoni E, Matteoli M: Synaptic and intrinsic mechanisms shape synchronous oscillations in hippocampal neurons in culture. Eur J Neurosci 1999, 11:389-397.

53. Dahlgren KN, Manelli AM, Stine WB Jr, Baker LK, Krafft GA, LaDu MJ: Oligomeric and fibrillar species of amyloid-beta peptides differentially affect neuronal viability. J Biol Chem 2002, 277:32046-32053.

doi:10.1186/1756-6606-3-10

Cite this article as: Rui et al: Inhibition of AMPA receptor trafficking at hippocampal synapses by $\beta$-amyloid oligomers: the mitochondrial contribution. Molecular Brain 2010 3:10.

\section{Submit your next manuscript to BioMed Central and take full advantage of:}

- Convenient online submission

- Thorough peer review

- No space constraints or color figure charges

- Immediate publication on acceptance

- Inclusion in PubMed, CAS, Scopus and Google Scholar

- Research which is freely available for redistribution

Submit your manuscript at www.biomedcentral.com/submit 